\title{
Fault Tolerant Design of Integrated Navigation Computer Based on SINS/CNS/GNSS
}

\author{
Ruimin Zhang ${ }^{1, a}$, Xusheng Wang ${ }^{2, b}$ and Shigang Fan ${ }^{3, c}$ \\ 1,2,3 High-tech Institute,Fan Gong-ting South Street on the 12th, QingZhou 262500 , China.
}

Keywords: Integrated navigation computer; fault-tolerant ability; hardware design; software design

\begin{abstract}
The target of the design of the integrated navigation computer based on SINS/CNS/GNSS is to improve the fault tolerance of navigation. In this paper, the design ideas are explained, and from the perspective of the hardware and software, the navigation is improved and supplemented, mainly aiming at the improvement of the fault-tolerant ability of the navigation computer. At the end of the paper, we compare the newly designed navigation system with the previous system. By contrast, we can know that the newly designed navigation system has certain practical significance.
\end{abstract}

\section{Introduction}

The integrated navigation computer system has the function that the traditional navigation system does not have, it can decompose the target in the navigation process and implement the navigation accurately. Also it allows different navigation information to complement and support each other to make navigation information more reliable, so many navigators prefer to use this technique in practice. However, the integrated navigation system is not perfect in every way. The integrated navigation system has a recognized shortcoming, that is, each part influences each other and the failure of some part can cause the paralysis of other parts[1]. For this reason, this paper designs the fault tolerance for integrated navigation computer in order to improve the overall function of integrated navigation computer.

\section{Introduction of Fault-tolerant Methods for Integrated Navigation Computers}

The SINS/CNS/GNSS system is mainly based on SINS, and the rest of it helps to achieve navigation goals. The navigation system obtains the required data and information from the integrated system to make the analysis, and shares them between the various parts of the system to realize the all-round control and guidance for the navigation system and synthetically analyze the geographical location, the surrounding information,etc. The aim of the design and research of the fault-tolerant ability of the integrated navigation computer is to improve the recognition capability of the computer, realize the timely self-repair of the navigation when it fails and achieve the fastest navigation in the shortest time. That is to say, the main function of the integrated navigation computer is to still be able to navigate while encountering problems, and will not affect navigation work. The main structure of the fault-tolerant navigation computer system is shown in figure 1 . 


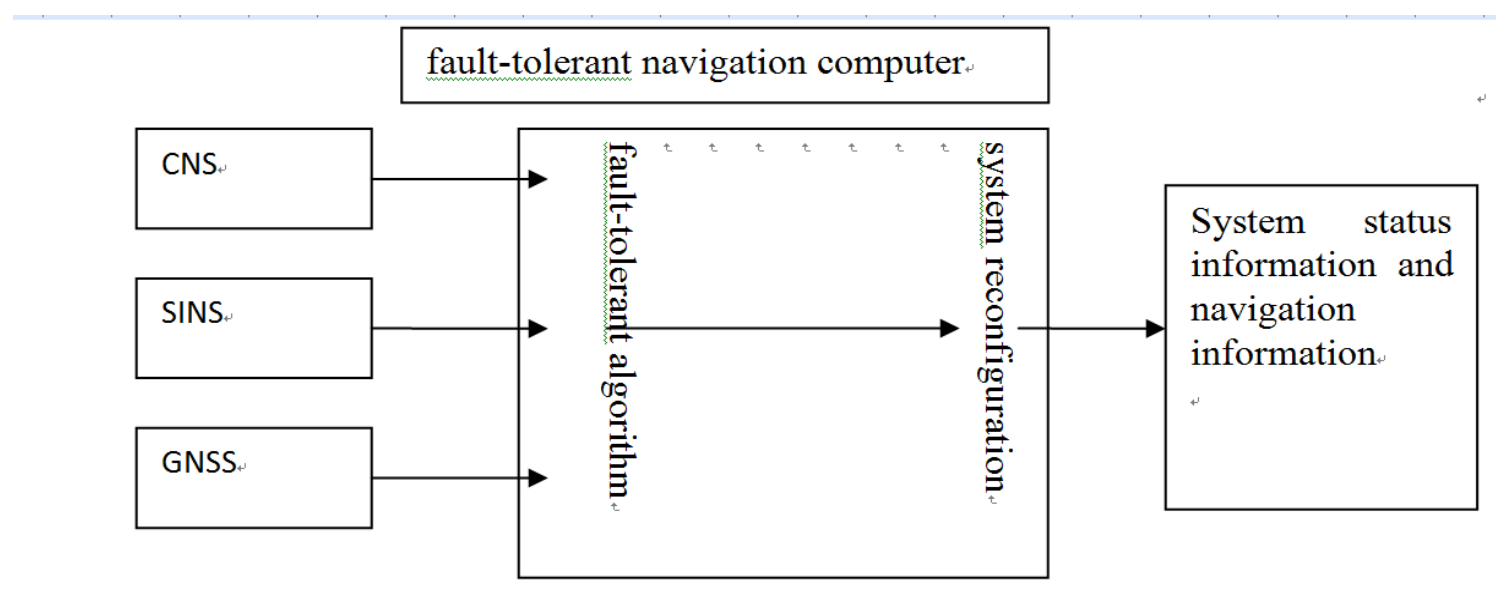

Figure 1. Structural schematic diagram of the fault-tolerant navigation computer system

The fault-tolerant design of the navigation computer can prompt the navigation system to do the timely self-repair while encountering the foreign invasion. According to the performance index that the computer can realize, the fault-tolerant algorithm is rebuilt, and the related factors of fault tolerance information and navigation are shown[2]. The fault-tolerant method of fault-tolerant integrated navigation computer can make the calculation of mathematical algorithm for navigation source and navigation system, recalculate the navigation according to the goal of navigation, and ensure the realization of the navigation target. The operational process of integrated navigation computer is often disturbed by various factors. The influence factor can be divided into hardware factor and software factor. As the name suggests, the hardware factors are mainly from the hardware system while the software factors are mainly from the software system. In order to improve the overall fault tolerance of navigation computer, this paper redesigned and constructed from these two factors.

\section{Hardware Fault Tolerance Design}

The fault-tolerant design of hardware is mainly to make the fault-tolerant design for the original or component part of the hardware of integrated navigation computer, or to make the backup design for the original which has the problem in the hardware component. And the negative effect of the error on the integrated navigation computer can be reduced to the minimum, and the navigation is ensured to not be affected by making up the defects of hardware[3]. Through the design of the computer hardware, we can ensure the realization of the navigation target. The performance of the different components of the integrated navigation computer is different, so the different designs are required.

No matter what kind of integrated navigation computer, they all need clock. Clock is an indispensable component of the integrated navigation computer system. Whether the clock is abnormal or not will directly affect the realization of the navigation function smoothly. The clock doesn't need a lot of energy, so the static redundancy fault-tolerant methods can be used to design it. Within the same time, different clocks in the integrated navigation computer system simultaneously release information to the system. In accordance with the running condition of the integrated navigation computer, the clock will automatically shield the clocks which have errors, don't accept the information from them, and prevent the error of the navigation because of the wrong information. Using the static redundancy fault tolerance method, it does not need to detect the function of the clock, nor does it need to rebuild the software of the clock. Clock can independently implement the fault-tolerant design by themselves. Using the static redundancy fault-tolerant design, each part of the clock consumes energy. The structure of the static redundancy clock is shown in figure 2 . 


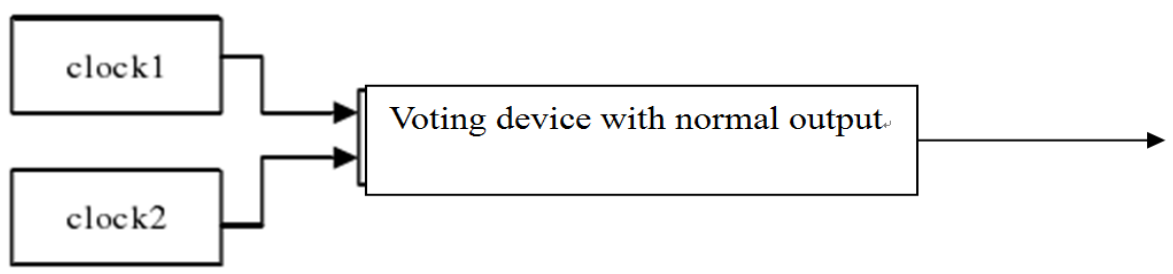

Figure 2. Structural schematic diagram of the static redundancy of clock

There are two kinds of redundant fault-tolerant design: static and dynamic. The structure of dynamic redundancy fault tolerance design is more complex, and the operational principle is also more complex. Its main working principle is to re-save the navigation information. When the operation of the system is abnormal, the newly saved information can be transferred to provide guidance for navigation and ensure the authenticity of the information source.

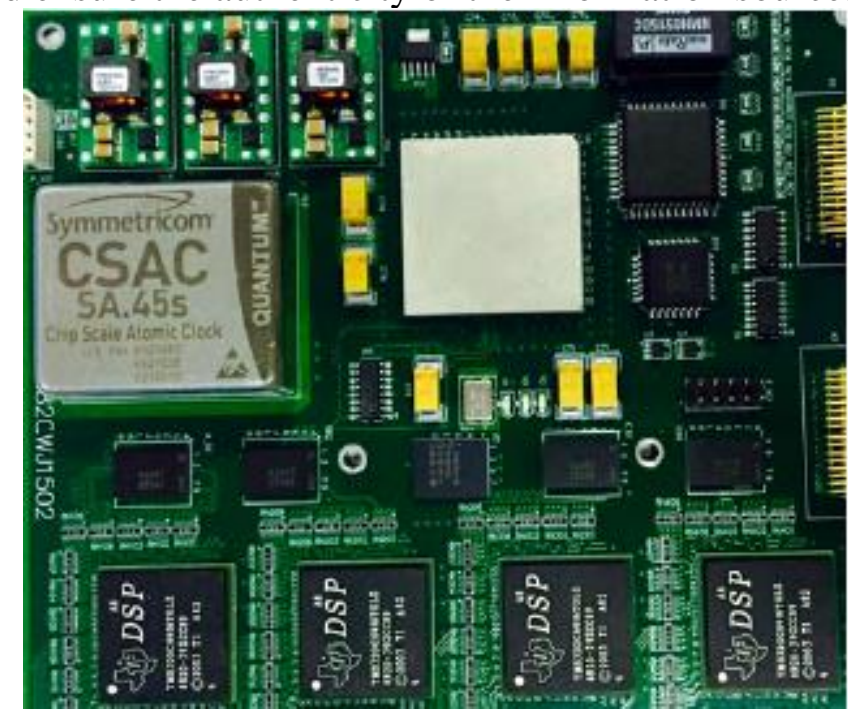

Figure 3. Local structure diagram of fault tolerant navigation computer

Hardware fault tolerance design can greatly improve the reliability of integrated navigation computer and realize the navigation target better.

\section{Software Fault Tolerance Design}

The design of software fault tolerance is mainly to make the fault tolerant design for the software part. The principal working method is to check the problems existing in computer software, check whether the algorithm of navigation is biased and ensure the realization of navigation destination. In general, the most common fault tolerant design for the software of integrated navigation computers is the use of $\mathrm{N}$ version programming[4]. This is a static fault repair method. Its main principle is to design multiple software with the same or similar function, and then put them into use simultaneously to calculate the navigation of integrated navigation computer and obtain the calculation results. $\mathrm{N}$ version programming has a strong adaptability and can be used in a variety of environments.

SINS/CNS/GNSS is three different navigation methods, and these three methods can be analyzed using an analysis program. The main purpose of the analysis is to deal with the redundant information of SINS. Different parts are responsible for the processing of different information, and the results of processing are transferred to the overall system. If the result of processing is not the same, then you can see that there is a fault in the system operation. At this time, you can decided whether to start the exception handling method[5]. Each module is a full-featured small processing system. The restoration and repair for the module is to make the backup for software information. Firstly, the module is put into use. The problems in the operation were detected in the use process. If fault is detected, we think that the test doesn't pass. The navigator can decide whether to restore the scene and start the backup system. 


\section{Comparison of Two Kinds of Integrated Navigation Computers}

Through the above discussion we find that the fault tolerant ability of previous navigation computer needs to be strengthened. We use two aspects of hardware and software to supplement, but whether the fault tolerant ability of the navigation computer supplemented in this paper in the actual navigation is effective still needs further verification. So in the following sections, we compare the practical work ability of the newly designed navigation computer with the previous ones.

Comparison of the Utilization Capacity of Navigation Source. For traditional integrated navigation computers, the processing procedures for their fault tolerance are approximately the same. They are one of the three ways of SINS/CNS/GNSS. According to the observation of traditional integrated navigation computer, we can find that when one of the three programs is out of order, it is likely to lead to the abnormal running of other program. The navigation location and surroundings can't be accurately determined. In the event of a failure, we can't timely determine which program is out of order. It will result in weaker utilization of the navigation source.

For the designed fault tolerant integrated navigation computer, the system can be analyzed timely when a problems occurs. We can determine which part of the system is out of order, and when we determine it, we can take corresponding measures for shielding the information from fault part and don't transmit it to the system. For the information and data provided by the abnormal navigation source, navigation system can't accept them. This is to ensure that the navigation process is not disturbed by the error information. The fault-tolerant computer will test the algorithm, and determine whether the operation of the algorithm has problems to ensure that the received information is correct. Therefore, from this aspect, the designed fault-tolerant integrated navigation computer has better utilization of navigation source. The following table reflects the available navigation information under different fault navigation sources.

Table1. Available navigation information under different fault navigation sources

\begin{tabular}{|c|c|c|c|}
\hline \multicolumn{3}{|c|}{ Navigation source working condition } & \multirow{2}{*}{$\begin{array}{c}\text { Available } \\
\text { navigation } \\
\text { information }\end{array}$} \\
\hline SINS & CNS & GNSS & \\
\hline normal & normal & normal & $\begin{array}{l}\text { Position, speed, } \\
\text { attitude, time and } \\
\text { other navigation } \\
\text { information are } \\
\text { output continuously } \\
\text { and reliably }\end{array}$ \\
\hline normal & normal & fault & $\begin{array}{l}\text { At this point, } \\
\text { GNSS should be } \\
\text { isolated by using } \\
\text { fault tolerant } \\
\text { algorithm. Only } \\
\text { SINS/CNS } \\
\text { integrated } \\
\text { navigation is used. } \\
\text { The accuracy } \\
\text { decreases when the } \\
\text { use time is long. }\end{array}$ \\
\hline normal & fault & normal & $\begin{array}{l}\text { At this point, CNS } \\
\text { should be isolated } \\
\text { by using fault } \\
\text { tolerant algorithm. } \\
\text { Only SINS/GNSS } \\
\text { integrated } \\
\text { navigation is used. }\end{array}$ \\
\hline
\end{tabular}




\begin{tabular}{|c|c|c|c|}
\hline & & & $\begin{array}{c}\text { The accuracy } \\
\text { decreases when the } \\
\text { use time is long. }\end{array}$ \\
\hline normal & fault & fault & $\begin{array}{l}\text { At this point, CNS } \\
\text { and GNSS should } \\
\text { be isolated by using } \\
\text { fault tolerant } \\
\text { algorithm. Only } \\
\text { SINS integrated } \\
\text { navigation is used. } \\
\text { When the time of } \\
\text { outputting } \\
\text { navigation } \\
\text { information is long, } \\
\text { the accuracy } \\
\text { decreases. }\end{array}$ \\
\hline fault & fault & fault & $\begin{array}{c}\text { Integrated } \\
\text { navigation system } \\
\text { can't be used. }\end{array}$ \\
\hline
\end{tabular}

Usability Comparison. The function of the previous navigation computer is not very perfect. If the operation of an analysis program is interrupted, the other programs will also become abnormal. From the actual effect, the usability of the previous navigation computer is not strong.

In this paper, the performance of the navigation computer is improved, and also the availability of navigation source is improved. Meanwhile, the usability is improved greatly. Fault tolerant integrated navigation computer has certain fault-tolerant ability. When a analysis program has an error, other programs can still continue to work. They can send correct and reliable navigation information to the overall system, and the overall system can rely on the normal analysis program to complete the navigation work. No matter which analysis program is out of order, the correctness of the information and data that eventually are transmitted to the overall system will not be affected. This will ensure the smooth completion of navigation.

\section{Conclusion}

In terms of the function, the previous integrated navigation computer could not fix the detected problems in time, which could affect the navigation results. However, this paper rebuilt the fault-tolerant ability of the integrated navigation computer, and from the perspective of the hardware and software, the performance of the navigation computer is improved. The fault-tolerant integrated navigation computer can shield and isolate the abnormal part. Through comparison, it is found that the fault-tolerant navigation computer not only improves the utilization capacity of navigation source, but also greatly improves the usability.

\section{References}

[1] J.Wang: Research on Fault-Tolerance Technology in Integrated Navigation Computer[J], Modern Navigation,2016,(01):24-27.

[2] L.F.Yang: Design and Implementation of Fault-tolerant Ruggedized Computer[D], Tianjin University, 2003.

[3] J.X.Liu and D.M.Huang: Design of the Computer System of a Fault Tolerant Integrated Navigation[J], Journal of Harbin Engineering University, 1999,(03):33-37.

[4] H.F.Li, Y.J.Li and W.Ning: SINS/CNS/GNSS Integrated Navigation System Based on CNS 
Simulator for Ballistic Missile[J], Journal of Projectiles, Rockets, Missiles and Guidance, 2008, 28(1):61-63.

[5] J.Wang: Research on SINS/CNS/GNSS Integrated Navigation Computer System[J], Modern Navigation, 2015(1):13-16. 